\title{
ON THE EQUIVALENT DESCRIPTIONS OF FAMILY OF FUNCTIONAL DEPENDENCIES IN THE RELATIONAL DATAMODEL.
}

\author{
VU DUC THI \\ Institute of Information Technology
}

\begin{abstract}
Summary. The family of functional dependencies (FDs) was introduced by E.F. Codd. Equivalent descriptions of family of FDs play essential rules in the design and implementation of the relation datamodel. It is known $[1,3,4,5,7,8,12,13,15]$ that closure operations, meet-semilattices, families of members which are not intersections of two other members give the equivalent descriptions of family of FDs. i.e. they and family of FDs determine each other uniquely. These equivalent description were successfully applied to find many desirable properties of functional dependency. This paper introduces the concept of maximal family of attributes. We prove that this family is an equivalent description of family of FDs. The concept of nonredundant family of attributes is also introduced in this paper. We present some charaterizations and desirable properties of these families.
\end{abstract}

\section{INTRODUCTION}

The relational datamodel which was introduced by E.F. Codd is one of the most powerful database models. The basic concept of this model is a relation. It is a table every row of which corresponds to a record and every column to an attribute. Because the structure of this model is clear, simple and mathematical instruments can be applied in it, it becomes the theoretical basis of database models. Semantic constraints between sets of attributes play very important roles in logical and structural investigations of relational datamodel both in practice and design theory. Important among these constraints is functional dependency. Equivalent description of family of FDs have been widely studied in the literature. Base on the equivalent descriptions we can obtain important properties of family of FDs. In this paper we investigate two families of sets. We show that one of them is an equivalent description of family of FDs. This paper give some results about computational problems related to these families. Let us give some necesarry definations and results that are used in next section. The concepts given in this section can be found in $[1,2,3,4,6,7,8,15]$.

Let $R=\left\{a_{1}, \ldots \ldots \ldots, a_{n}\right\}$ be a nonempty finite set of attributes. A functional dependency is a statement of the form $A \rightarrow B$, where $A, B \subseteq R$. The FD $A \rightarrow B$ holds in a relation $r=\left\{h_{1}, \ldots h_{m}\right\}$ over $R$ if $\forall h_{i}, h_{j} \in r$ we have $h_{i}(a)=h_{j}(a)$ for all $a \in A$ implies $h_{i}(b)=h_{j}(b)$ for all $b \in B$. We also say that $\mathrm{r}$ satisfies the FD $A \rightarrow B$.

Let $F_{r}$ be a family of all FDs that hold in $r$. Then $F=F_{r}$ satisfies. 
(1) $A \rightarrow A \in F$,

(2) $(A \rightarrow B \in F, B \rightarrow C \in F) \Rightarrow(A \rightarrow C \in F)$,

(3) $(A \rightarrow B \in F, A \subseteq C, D \subseteq B) \Rightarrow(C \rightarrow D \in F)$,

(4) $(A \rightarrow B \in F, C \rightarrow D \in F) \Rightarrow(A \cup C \rightarrow B \cup D \in F)$.

A family of FDs satisfying (1)-(4) is called an f-family (sometimes it is called the full family) over $R$.

Clearly, $F_{r}$ is an f-family over $R$. It is known [1] that if $F$ is an arbitrary f-family, then there is a relation $r$ over $R$ such that $F_{r}=F$.

Given a family $F$ of FDs, there exits a unique minimal f-family $F^{+}$that contains $F$. It can be seen that $F^{+}$contains all FDs which can be derived from $F$ by the rules (1)-(4).

A relation schemes is a pair $\langle R, F\rangle$, where $R$ is a set of attributes, and $F$ is a set of FDs over $R$. Denote $A^{+}=\left\{a: A \rightarrow\{a\} \in F^{+}\right\} . A^{+}$is called the closure of $A$ over $s$. It is clear that $A \rightarrow B \in F^{+}$iif $B \subseteq A^{+}$.

Clearly, if $s=\langle R, F\rangle$ is a relation scheme, then there is a relation $r$ over $R$ such that, $F_{r}=F^{+}$(see, [1]). Such a relation is called an Amstrong relation of $s$.

Let $R$ be a nonempty finity set of attributes and $P(R)$ its power set. The mapping$H: P(R) \rightarrow P(R)$ is called a closure operation over $R$ if for all $A, B \in P(R)$, the following conditions are satisfied:

(1) $A \subseteq H(A)$,

(2) $A \subseteq B$ implies $H(A) \subseteq H(B)$,

(3) $H(H(A))=H(A)$

Let $s=\langle R, F\rangle$ be a relation scheme. Set $H_{s}(A)=\left\{a: A \rightarrow\{a\} \in F^{+}\right\}$, we can see that $H_{0}$ is a closure operation over $R$.

Let $r$ be a relation, $s=\langle R, F\rangle$ be a relation scheme. Then $A$ is a key of $r$ (a key of s) if $A \rightarrow R \in F_{\mathrm{r}}\left(A \rightarrow R \in F^{+}\right)$. A is a minimal key of $r(s)$ if $A$ is a key of $r(s)$ and any proper subset of $A$ is not a key of $r(s)$.

Denote $K_{r}\left(K_{s}\right)$ the set of all minimal keys of $r(s)$, i.e. $A, B \in K_{r}$ implies $A \nsubseteq B$.

Clearly, $K_{\mathrm{r}}, K_{\mathrm{s}}$ are Sperner systems over $R$.

Let $K$ be a Sperner system over $R$. We define the set of antikeys of $K$, denoted by $K^{-1}$, as follows:

$$
K^{-1}=\{A \subset R:(B \in K) \Rightarrow(B \nsubseteq A) \text { and }(A \subset C) \Rightarrow(\exists B \in K)(B \subseteq C)\}
$$

It is easy to see that $K^{-1}$ is also a Sperner system over $R$. 
It is known [5] that if $K$ is an arbitrary Sperner system over $R$, then there is a relation scheme such that $K_{s}=K$.

In this paper we always assume that if a Sperner system plays the role of the set of minimal keys (antikeys), then this Sperner system is not empty (doesn't contain $R$ ). We consider the comparision of two attributes as an elementary step of algorithms. Thus, if we assume that subset of $R$ are represented as sorted lists of attributes, then a Boolean operation on two subsets of $R$ requires at most $|R|$ elementary steps.

Let $L \subseteq P(R) . L$ is called a meet-irreducible family over $R$ (sometimes it is called a family of members which are not intersection of two other members) if $\forall A, B, C \in L$, then $A=B \cap C$ implies $A=B$ or $A=C$.

Let $I \subseteq P(R), R \in I$, and $A, B \in I \Rightarrow A \cap B \in I$ (Sometimes $I$ is called a meetsemilattice over $R$ ). Let $M \subseteq P(R)$. Denote $M^{+}=\left\{\cap M^{\prime}: M^{\prime} \subseteq M\right\}$. We say that $M$ is a generator of $I$ if $M^{+}=I$. Note that $R \subseteq M^{+}$but not in $M$, by convention it is the intersection of the empty collection of sets.

Denote $N=\left\{A \in I: A \neq \cap\left\{A^{\prime} \in I: A \subset A^{\prime}\right\}\right\}$.

In [5] it is proved that $N$ is the unique minimal generator of $I$.

It can be seen that $N$ is a family of members which are not intersections of two other members.

Let $H$ be a closure operation over $R$. Denote $Z(H)=\{A: H(A)=A\}$ and $N(H)=$ $\left\{A \in Z(H): A \neq \cap\left\{A^{\prime} \in Z(H): A \subset A^{\prime}\right\}\right\} . Z(H)$ is called the family of closed sets of $H$. We say that $N(H)$ is the minimal generator of $H$.

It is shown [5] that if $L$ is a meet-irreducible family then $L$ is the minimal generator of some closure operation over $R$. It is known [1] that there is an on-to-one correspondence between these families and f-family.

Let $r$ be a relation over $R$. Denote $E_{r}=\left\{E_{i j}: 1 \leq i<j \leq|r|\right\}$, where $E_{i j}=\{a \in R$ : $\left.h_{i}(a)=h_{j}(a)\right\}$. Then $E_{\mathrm{r}}$ is called the equality set of $r$.

Let $T_{r}=\left\{A \in P(r): \exists E_{i j}=A, A E_{p q}: A \subset E_{p q}\right\}$. We say that $T_{r}$ is the maximal equality system of $r$.

Let $r$ be a relation, and $K$ a Sperner system over $R$. We say that $r$ represents $K$ if $K_{\mathrm{r}}=K$.

The following theorem is known ([8]).

Theorem 1.1. Let $K$ be a non-empty Sperner system and $r$ a relation over $R$. Then $r$ represents $K$ iff $K^{-1}=T_{r}$, where $T_{r}$ is the maximal equality system of $r$.

Let $s=\langle R, F\rangle$ be relation scheme over $R, K$, is a set of all minimal keys of $s$. Denote 
by $K_{s}^{-1}$, the set of all antikeys of $s$. From Theorem 1.1 we obtain the following corollary.

Corollary 1.2. Let $s=\langle R, F\rangle$ be a relation scheme and $r$ a relation over $R$. We say that $r$ represents if $K_{r}=K_{\theta}$. Then $r$ represents $s$ iff $K_{s}^{-1}=T_{r}$, where $T_{r}$ is the maximal equality system of $r$.

In [7] we proved the following theorem.

Theorem 1.3. Let $r=\left\{h_{1}, \ldots, h_{m}\right\}$ be a relation, and $F$ an f-family over $R$. Then $F_{r}=F$ iff for every $A \subseteq R$

$$
H F(A)= \begin{cases}\cap_{A \subseteq E_{i j}} E_{i j}, & \text { if } \exists E_{i j} \in E_{r}: A \subseteq E_{i j} \\ R, & \text { otherwise. }\end{cases}
$$

Where $H_{F}(A)=\{a \in R: A \rightarrow\{a\} \in F\}$ and $E_{r}$ is the equality set of $r$.

Theorem 1.4. [4] Let $K$ be a Sperner system and $s=\langle R, F\rangle$ a relation scheme over $R$. Denote $Z(s)=\left\{A: A^{+}=A\right\}$. Then $K_{0}=K$ iff $\{R\} \cup K^{-1} \subseteq Z(s) \subseteq\{R\} \cup G\left(K^{-1}\right)$, where $G\left(K^{-1}\right)=$ $\left\{A: \exists B \in K^{-1}: A \subseteq B\right\}$.

Clearly, (see [4]) we have

Theorem 1.5. Let $K=\left\{K_{1}, \ldots ., K_{m}\right\}$ be a Sperner system over $R$. Set $s=\langle R, \bar{F}\rangle$ with $F=\left\{K_{1} \rightarrow R, \ldots, K_{m} \rightarrow R\right\}$. Then $K_{s}=K$ and $Z(s)=G\left(K^{-1}\right) \cup\{R\}$.

\section{RESULTS}

In this section we introduce the concept of maximal family of attributes. We show that this family and family of FDs determine each other uniquely. We give some desirable properties of this family. We also introduce the concept of nonredundant family of attributes. We present some results about connections between this family, meet-irreducible family and closure operation.

Now we introduce the following concept.

Definition 2.1. Let $R$ be a nonempty finite set of attributes. A family $M=\{(A,\{a\}): A \subset$ $R, a \in R\}$ is called a maximal family of attribute over $R$ iff the following conditions are satisfied:

(1) $a \notin A$,

(2) For all $(B,\{b\}) \in M, a \in B$ and $A \subseteq B$ imply $A=B$.

(3) $A(\dot{B},\{b\}) \in M: a \notin B, a \neq b$, and $L_{a} \cup B$ is a Sperner system over $R$, where $L_{a}=\{A:(A,\{a\}) \in M\}$. 


\section{Remark 2.2.}

$(-)$ It is possible that there are $(A,\{a\}),(B,\{b\}) \in M$ such that $a \neq b$, but $A=B$.

$(-)$ It can be seen that by (1) and (2) for each $a \in R L_{a}$ is a Sperner system over $R$. It is possible that $L_{a}$ is an empty Sperner system.

$(-)$ Let $R$ be a nonempty finite set of attribute and $P(R)$ its power set. According to definition 2.1 we can see that given a family $Y \subseteq P(R) \times P(R)$ there is a polynomial time algorithm deciding whether $Y$ is a maximal family of attribute over $R$.

Let $H$ be a closure operation over $R$. Denote $Z(H)=\{A: H(A)=A\}$ and $M(H)=$ $\{(A,\{A\}): a \notin A, A \in Z(H)$ and $B \in Z(H), A \subseteq B, a \notin B$ imply $A=B\}$.

$Z(H)$ is called the family of closed sets of $H$. It can be seen that for each $(A,\{a\}) \in$ $M(H) A$ is a maximal closed set which doesn't contain $a$.

It is possible that there are $(A,\{a\}),(B,\{b\}) \in M_{(}(H)$ such that $a \neq b$, but $A=B$.

Remarks 2.3. Let $R$ be a relation over $R$ and $F_{r}$ a family of all FDs that hold in $r$. Denote $A_{r}^{+}=\left\{a: A \rightarrow\{a\} \in F_{r}\right.$. Set $Z_{r}=\left\{A: A=A_{r}^{+}\right\}$. Denote by $N_{r}$ the minimal generator of $Z_{\mathrm{r}}$. It can be seen that $N_{\mathrm{r}} \subseteq E_{\mathrm{r}}$ and $N_{r}=\left\{A \in E_{\mathrm{r}}: A \neq \cap\left\{B: B \in E_{\mathrm{r}}, A \subset B\right\}\right.$, where $E_{\mathrm{r}}$ is the equality set of $r$.

We give the following theorem which shows that closure operations and maximal families of attributes determine each other uniquely.

Theorem 2.4. Let $H$ be a closure operation over $R$. Then $M(H)$ is a maximal family of attributes over $R$. Conversely, if $M$ is a maximal family of attributes over $R$ then there exits exactly one closure operation $H$ over $R$ so that $M(H)=M$, where for all $B \in P(R)$.

$$
H(B)= \begin{cases}\cup_{B \subseteq A} A, & \text { if } \exists A \in L(M): B \subseteq A \\ R, & \text { otherwise }\end{cases}
$$

and $L(M)=\{A:(A,\{a\}) \in M\}$.

Proof: Assume that $H$ is a closure operation over $R$. Based on the definition of $M(H)$ we have (1) and (2). We set $L_{a}^{\prime}=\{A:(A,\{a\} \in M(H)\}$. Suppose that there is a $(B,\{b\}) \in M(H): a \neq b, a \notin B, L_{a}^{\prime} \cup B$ is a Sperner system over $R\left(^{*}\right)$. Then we choose $(B,\{b\}) \in M(H)$ such that $B$ is maximal for $\left(^{*}\right)$. By $(2)$ in Definition 2.1 we see that $L^{p}$ rime $e_{a}$ is a Sperner system over $R$. Consequently, there is no an $A \in L_{a}^{\prime}$ such that $B \subseteq A$. Accorrding to the definition of $M(H)$ we have $(B,\{a\}) \in M(H)$. Thus, $B \in L_{a}^{\prime}$ holds. This is a constradiction. Hence, we have (3) in Definition 2.1 i.e. $M(N)$ is a minimal family of attributes over $R$. Conversely, assume that $M$ is a maximal family of attributes 
over $R$. Denote $L(M)=\{A:(A,\{a\}) \in M\}$. First we will prove that $L(M)$ is a meetirreducible family over $R$. For any $(A,\{a\}) \in M$ by Remark 2.2 we have $A \neq A^{\prime} \cap A^{\prime \prime}$ and $A \neq A^{\prime} \cap B$, where $A^{\prime}, A^{\prime \prime} \in L_{a}$ and $B \in L(M): A \neq B$. If there are $(B,\{b\}),(C,\{c\}) \in M$ such that $b \neq a, c \neq a, A \subset B, A \subset C$ then by (2) in Definition 2.1 we have $a \in B, a \in C$. Hence, $A \subset B \cap C$ holds. Thus, for all $A, B, C \in L(M)$ if $A=B \cap C$ then $A=B$ or $A=C$. Consequently, $L(M)$ is a meet-irreducible family over $R$. It is known[5] that meetirreducible families and closure operations determine each other uniquely. On the other hand, according to Remark 2.3 and Theorem 1.3 we can see that $H$ is a closure operation over $R$, and $L(M)$ is the minimal generator of $Z(H)$.

Now we have to prove that $M(H)=M$. If $(A,\{a\}) \in M$ then $A \in L(M)$. Suppose that for each $b \neq A$ there exists a $B \in Z(H): A \subset B, b \notin B$. It can be seen that $A$ is the intersection of such $B s$. This conflicts with the fact that $A \in L(M)$. Thus, if $(A,\{a\}) \in M$ then there is a $b \notin A$ such that $(A,\{b\}) \in M(H)\left({ }^{* *}\right)$. If $(A,\{a\}) \in M(H)$ then according to definition of $M(H) B \in Z(H)$, and $A \subset B$ imply $a \in B$. By $a \notin A$ we can see that $A$ is not the intersection of such $B$. According to construction of $H$ we have $A \in L(M)$. Thus, if $(A,\{a\}) \in M(H)$ then $A \in L(M)\left(^{* * *}\right)$. Now suppose that $(A,\{a\}) \in M$, but $(A,\{a\}) \in M(H)$. Because $A$ is a closed set of $H, a \in A$ and by definition of $M(H)$ there is a $(B,\{a\}) \in M(H)$ such that $A \subset B$. By $\left(^{* * *}\right) B \in L(M)$ holds. This conflicts with condition (2) of Definition 2.1. Hence 2.1, $(A,\{a\}) \in M(H)$ holds.

Suppose that $(A,\{a\}) \in M(H)$, but $(A,\{a\}) \notin M$. We consider $L_{a}$. If there is an $A^{\prime} \in L_{a}$ such that $A \subset A^{\prime}$ then by $\left({ }^{* *}\right)$ we can see that $A^{\prime}$ is closed set of $H$. Accorrding to definition $M(H)$ we obtain $(A,\{a\}) \in M(H)$. This is a contracxdiction. If $A^{\prime} \subset A$ then by $\left({ }^{* * *}\right)$ we can see that this conflicts with condition (3) of Definition 2.1. Consequently, there is an $A^{\prime} \in L_{a}$ so that $A=A^{\prime}$. Hence, $M(H)=M$ holds.

If we suppose that there is a closure operation $H^{\prime}$ such that $M\left(H^{\prime}\right)=M$. Denote $L\left(H^{\prime}\right)=\left\{A:(A a \in M(H)\}\right.$. According to parts $\left({ }^{* *}\right)$ and $\left({ }^{* *}\right)$ of above proof we can see that $L\left(H^{\prime}\right)$ is the minimal generator of $Z\left(H^{\prime}\right)$. By $M\left(H^{\prime}\right)=M(H)=M$ we have $L\left(H^{\prime}\right)=L(M)=L(H)$. Because closure operations and meet-irreducible families determine each other uniquely, we obtain $H=H^{\prime}$. The proof complete.

\section{$\because$}

It is known [5] that closure operation and families of FDs determine each other uniquely anf from Theorem 2.4 the fo 2.4 the following corollary is clear:

Corollary 2.5. There exists an one-to-one correspondence between maximal families of attributes anf families of FDs.

Based on the proof of Theorem 2.4 we have the following. 
Propositon 2.6. Let $H$ be a closure operation over $R$. Denote $Z(H)=\{A: H(A)=A\}$ and $M(H)=\{(A,\{a\}): a(A, A(Z(H)$ and $B \in Z(H), A \subset B, a \in B$ imply $A=B\}$. Set $L(H)=\{A:(A,\{a\}) \in M(H)\}$. Then $L(H)$ is the minimal generator of $Z(H)$, i.e. it is also the minimal generator of $H$.

Remark 2.7. It is known [11] that if $s=<R, F>$ is a relation scheme, $\operatorname{denote} Z(s)=\{A$ : $\left.A^{+}=A\right\}$, and $N_{s}$ is a minimal generator of $Z(s)$, then

$$
N_{s}=\operatorname{MAX}\left(F^{+}\right)=\cup_{a \in R} M A X\left(F^{+}, a\right),
$$

where

$$
M A X\left(F^{+}, a\right)=\left\{A \subseteq R: A \rightarrow\{a\} \notin F+, A \subset B \Rightarrow B \rightarrow\{a\} \in F^{+}\right\}
$$

Proposition 2.8. Let $F$ be an family over $R, a \in R$. Dengte $L F(A)=\{a \in R: A \rightarrow\{a\} \in$ $F\}, Z F=\{A: L F(A)=A\}$. Clearly, $R \in Z F$, and $Z F$ closed under intersection. Denote by $N F$ the minimal generator of $Z F$. Set $M_{a}=\left\{A \in N_{F}: a \notin A, A B \in N_{F}: a \notin B, A \subset B\right\}$. Then $M_{a}=\operatorname{MAX}(F, a)$, where $M A X(F, a)=\{A \in R: A$ is a nonempty maximal set such that $(A,\{a\}) \notin F\}$.

Proof: It is known [11] that $M A X(F, a) \subseteq N_{F}$ holds (1). Assume that $A \in M_{a}$. By $A \in N_{F}$, i.e. $L_{F}(A)=A$ and $a \in A$ we obtain $(A,\{a\}) \in F$. From (1) and according to the definition of $M_{a}$ we have $A \in M A X(F, a)$. Conversely, if $A \in M A X(F, a)$ then by (1) $A \in N_{F}$ holds (2). By $(A,\{A\}) \notin F$ and from (2) we obtain $a \notin A$. According to the definition of $M A X(F, a)$ we have $A \in M_{a}$. Our proof is complete.

It is known [1] that meet-irreducible families and meet-semilattices detemine each other uniqurly. On the other hand, from Proposition 2.8 and based on definition of $M(H)$ and Definition 2.1 the next corollary is clear.

Corollary 2.9. Let $s=<R, F>$ be a relation scheme. Then $\left\{(A,\{a\}): A \in M A X\left(F^{+}, a\right), a \in\right.$ $R\}$ is a maximal family of attributes over $R$.

It is known [9] that we used the family $\left\{(A,\{a\}): A \in M A X\left(F^{+}, a\right), a \in R\right\}$ in algorithm for generating Armstrong relations and inferring functional dependencies.

It can be seen that in definition of maximal family $M$ attributes over $R$ it is possible that there are $(A,\{a\}),(B,\{b\}) \in M$ such that $a \neq b$, but $A=B$. We introduce the new concept which deletes redundancy.

Definition 2.10. Let $R$ be a nonempty finite set of attribute. A family $M=\{(A,\{a\}): A \subset$ $R, a \in R\}$ is called a nonredundant family of attribute over $R$ if for all $(A,\{a\}),(B,\{b\}) \in M$ : 
(1) $a \notin A$.

(2) $a \notin B$ and $A \subseteq B$ imply $A=B$.

(3) $\nexists B,\{b\}) \in M: a \neq b, A=B$.

It can be seen that for each $a \in R L_{a}=\{A:(A,\{a\}) \in M\}$ is a Sperner system over $R$. It is possible that $L_{a}$ is an empty Sperner system. It is easy to see that given a family $Y \subseteq P(R) \times P(R)$ there is a polynomial time algorithm deciding whether $Y$ is a nonredundant family of attribute over $R$. Definition 2.11. Let $H$ be a closure over $R$. Denote $Z(H)=\{A: H(A)=A\}$. Then we say that the set $T=\{(A,\{a\}: a \in A, A(Z(H)\}$ is minimal of $H$ if

$\left(^{*}\right) \nexists B \in Z(H): A \subset B, a \notin B$

$\left(^{* *}\right) \nexists(B,\{b\}) \in T: A=B, a \neq b$.

It can be seen that $T$ is a minimal set of $H$ then $T \subseteq M(H)$. It is possible that for any closure operation $H$ there are many minimal sets of $H$. However, we have the next lemma.

Lemma 2.12. Let $H$ be a closure operation over $R$. $T$ a minimal set of $H$. Denote by $N(H)$ the minimal generator of $H$. Set $L(T)=\{A:(A,\{a\}) \in T\}$, Then $L(T)=N(H)$ and $|N(H)|=|T|$.

Proof. Denote by $N(H)$ the minimal generator of $H$, i.e. $N(H)=\left\{A \in Z(H): A \neq \cap\left\{A^{\prime} \in\right.\right.$ $\left.\left.Z(H): A \subseteq A^{\prime}\right\}\right\}$. We have to prove that $L(T)=N(H)$. Assume that $A \in L(T)$. By $\left(^{*}\right)$ in definition 2.11 we can see that if $B \in Z(H)$ and $A \subset B$ then $a \in B$. By $a \notin A A$ is not the intersection of such $B s$. It is obvious that $A \in Z(H)$. Hence, $A \in N(H)$ holds. Concersely, assume that $A \in N(H)$. Suppose that for each $b \notin A$ there exits a $B \in Z(H): A \subset B, b \notin B$. It can be seen that $A$ is the intersection of such $B s$. This conflicts with the fact that $A \in N(H)$. By $\left({ }^{* *}\right)$ in definition of $T$ we see that the number of elements of $T$ is $|N(H)|$. The lemma is proved.

Based on Lemma 2.12, Definition 2.11 and definition of $M(N)$ we have the following.

Corollary 2.13. Let $H$ be a closure operation over $R$. Denote by $N$ the minimal generator of $H$. Then $H$ has an unique minimal set iff $|N|=|M(H)|$.

Based on Lemma 2.12, Definition 2.11, 2.10 and according to Lemma 2.12 the next corollary is clear.

Corollary 2.14. Let $M$ be a maximal family of attribute over $R$. Denote $L(M)=\{A$ : $(A,\{a\}) \in M\}$. Then $M$ is a nonredundant family of attribute iff $|M|=|L(M)|$ holds.

Based on Lemma 2.12 we give the following. 
Theorem 2.15. Let $H$ be a closure operation over $R, T$ a minimal set of $H$. Then $T$ is a nonredundant family of attributes over $R$. Conversely, if $M$ is a nonredundant family of attribute over $R$ then there exits exactly one closure operation $H$ over $R$ such that $M$ is a minimal set of $H$, where for all $C \in P(R)$

$$
H(C)= \begin{cases}\cap_{C \subseteq A} A, & \text { if } \exists A \in L(M): C \subseteq A \\ R, & \text { otherwise. }\end{cases}
$$

and $L(M)=\{A:(A,\{a\}) \in M\}$.

Proof. According to Definitions 2.10 and 2.11 it is obvious that if $T$ is a minimal set of a given closure operation then $T$ is a nonredundant family of attributes over $R$. Conversely, if $M$ is a nonredundant family of attributes over $R$ then by the proof of Theorem 2.4 we can see that $L(M)$ is a meet-irreducible family over $R$, and $L(M)$ is the minimal generator of $Z(H)$, i.i. $L(M)=N(H)$. we have to prove that $M$ is a minimal set of $H$. It can be seen that by construction of $\mathrm{H}$, if $(A,\{a\}) \in M$ then $a \notin A$ and $A$ is a closed set of $H$. Clearly, $M$ satisfies the condition $\left({ }^{* *}\right)$ of Definition 2.11. Suppose that there exits a $B \in Z(H)$ such that $a \in B$ and $A \subset B(1)$. If $B \in N(H)$ then by $L(M)=N(H)$ we can sce that (1) conflicts with (2) in Definition 2.10. If $B \in\{C: C \in L(M), B \subset C\}$ then by $a \notin B$ we can see that there is a such $C$ so that $a \notin C$. This is a contracdiction. Consequently, $M$ satifies the condition $\left(^{*}\right)$ in DEfinition 2.11. Thus, $M$ is a minimal set of $H$. Now we suppose that there is a closure operation $\mathrm{H}^{\prime}$ such that $M$ is also a minimal set of $H^{\prime}$. According to Lemma 2.12 we obtain $L(M)=N\left(H^{\prime}\right)$, where $N\left(H^{\prime}\right)$ is the minimal generator of $H^{\prime}$. Hence, $N(H)=N\left(H^{\prime}\right)$ holds. Because closure operations and meet-irreducible families determine each other uniquely. We obtain $H=H^{\prime}$. The theorem is proved.

Now we give the following example.

Example 2.16. $r$ is the following relation over $R=\{a, b, c, d\}$ :

$\begin{array}{cccc}\mathrm{a} & \mathrm{b} & \mathrm{c} & \mathrm{d} \\ 0 & 0 & 2 & 2 \\ 5 & 0 & 0 & 7 \\ 1 & 1 & 0 & 0 \\ 0 & 0 & 0 & 0 \\ 3 & 3 & 3 & 0\end{array}$

It is easy to see that

$E_{\mathrm{r}}=\{\{a, b\},\{b, c\},\{c, d\},\{b\},\{c\},\{d\}, 0\}$. 
By remark 2.3 we have $N_{r}=\{\{a, b\},\{b, c\},\{c, d\},\{d\}\}$. Clearly, $F_{r}$ is an f-family over $R$. Denote $L_{F_{r}}(A)=\{a: A \rightarrow\{a\}\}$. It can be seen that $L_{F_{r}}$ is a closure operation over $R$ and $N_{r}$ is the minimal generator of $L_{F_{r}}$ it also is the minimal generator of $Z_{F_{r}}$. According to Propositition 2.S we obtain

$$
\begin{aligned}
& M_{a}=\{\{b, c\},\{c, d\}\}, M_{b}=\{c, d\}, \\
& M_{c}=\{\{a, b\},\{d\}\}, M_{d}=\{\{a, b\},\{b, c\}\} .
\end{aligned}
$$

By Proposition 2.8 and Corollary 2.9

$$
\begin{aligned}
& M\left(L_{F_{r}}\right)=\{(\{b, C\},\{a\}),(\{c, d\},\{a\}),\{c, d\},\{b\}) . \\
& (\{a, b\},\{c\}),\{d\},\{c\}),(\{a, b\},\{d\}),(\{b, c\},\{d\})\}
\end{aligned}
$$

is the maximal family of attributes over $R$.

According to Definition 2.11 we obtain sọme minimal sets of $L_{F_{r}}$ as follows:

$$
\begin{aligned}
& T_{1}=\{(\{b, c\},\{a\}\},\{c, d\},\{b\},(\{a, b\},\{c\},(\{d\},\{c\})\}, \\
& T_{2}=(b, c\},\{a\},\{c, d\},\{a\},(\{d\{c\},(\{a, b\},\{d\})\},
\end{aligned}
$$

Clearly, they are nonredundant families of attribute over $R$ and $L\left(T_{1}\right)=L\left(T_{2}\right)=$ $L(M)=N_{r}$.

\section{REFERENCES}

1. Armstrong W.W., Dependency Structures of Database Relationships, Information Processing 74, Holland Publ. Co. (1974) pp. 580-583.

2. Beeri C., Bernstein P.A., Computational problems related to th design of normal form relation schemes, ACM Trans. on Database Syst. 4,1 (1979) pp. 30-59.

3. Bekessy A., Demetrovics J., Contribution to the theory of data base relations, Discrete Math. 27 (1979) pp,. 1-10.

4. Burosch G., Demetrovics J., Katona G.O.H., The poset of closures as a model of changing databases, Order 4 (1987) pp. 127-142.

5. Demetrovics J., Logical and structural investigation on relational datamodel (in Hungarian), MTA-SZTAKI Tanulmanyok, Budapest, 114 (1980) pp. 1-97.

6. Demetrovics J., Thi V.D., Some results about functional dependencies, Acta Cybernetica 8,3 (1988) pp. 273-278.

7. Demetrovics J., Thi V.D., Relation and minimal keys, Acta Cybemetica 8,3 (1988) pp. 279-285. 
8. Demetrovics J., Thi V.D., On Keys in the Relational Datamodel, J. Inform. Process. Cybernet. 24 (1988) 10, pp. 515-519.

9. Demetrovics J., Thi V.D., On algorithms for generating Armstrong relations and inferring functional dependencies in the Relational datamodel, Computers and Mathematics with Applications, British Vol 26, No 4 (1993), pp. 43-55.

10. Demetrovics J., Thi V.D., Armstrong Relation, Functional Dependencies and Strong Dependencies, Comput and AI ( to appear).

11. Manila H., Raiha K.J., Design by Example: An Application of Armstrong relations, J. Comput. Syst. Scien 33 (1986) pp. 126-141.

12. Thi V.D., Investigation on Combinatorial Characterzations Related to Functional Dependency in the Relational Datamodel, MTA SZTAKI Tanulmanyok, Budapest, 191 (1986) pp.1-157.

13. Thi V.D., Minimal Keys and Antikeys, Acta Cybemetica 7, 4 (1986) pp. 361-371.

14. Thi V.D., On Antikeys in the Relational Datamodel (in Hungarian), Alkamazott Matamatikai Lapok 12 (1986) pp. 111-124.

15. Thi V.D., Logical Dependencies and irrdundant relations, Computers and Artifical Intelligent, 7 (1988) pp. 165-184. 